\title{
Imaging of the Cardiac Sympathetic Nervous System Has Potential Value in the Evaluation of Patients with Heart Failure with Preserved Ejection Fraction
}

\author{
Nabil E. Boutagy ${ }^{1}$ and Albert J. Sinusas ${ }^{1,2}$ \\ ${ }^{1}$ Section of Cardiovascular Medicine, Department of Internal Medicine, Yale University School of Medicine, New Haven, \\ Connecticut; and ${ }^{2}$ Department of Radiology and Biomedical Imaging, Yale University School of Medicine, New Haven, Connecticut
}

\section{See the associated article on page 784 .}

H eart failure (HF) is a major public health problem that affects more than 5.8 million people in the United States and 23 million people worldwide (1). HF is a clinical syndrome, rather than a disease, and can occur in patients with reduced and preserved left ventricular (LV) ejection fraction. Community-based studies indicate that approximately $50 \%$ of patients with the clinical diagnosis of HF have preserved EF (HFpEF), whereas the remaining patient population presents with HF with reduced EF (HFrEF) $(2,3)$. The values used to define preserved EF range in the literature from $40 \%$ to $55 \%$, but current guidelines recommend an $\mathrm{EF}$ of more than $50 \%$ with elevated natriuretic peptide (BNP or NTproBNP) levels and relevant structural heart disease, such as left atrial enlargement or LV diastolic dysfunction, as criteria for HFpEF (4,5). Although clinical symptoms and mortality are similar among patients with HFpEF and HErEF, there are pronounced differences between these HF phenotypes in patient demographics, responses to therapy, and underlying pathophysiology of LV remodeling $(6,7)$. Importantly, the prevalence of $\mathrm{HFpEF}$ relative to $\mathrm{HFrEF}$ is rising at a rate of $1 \%$ per year and will thus dominate as the prevalent HF phenotype over the next decade (8). Despite the rising prevalence, there are limited data to support effective therapies for $\mathrm{HFpEF}$, and the role of diagnostic strategies and prognostic biomarkers remain ambiguous $(4,9)$.

Excessive cardiac sympathetic nervous system (SNS) activation is a hallmark of $\mathrm{HF}$ progression in patients with $\mathrm{HFrEF}(10-12)$. A compensatory increase in adrenergic drive causes desensitization/ downregulation of the norepinephrine transporter (or uptake-1 mechanism) on the cardiac presynaptic nerve terminal due to excess norepinephrine in the synaptic cleft. The downregulation of uptake-1 exposes the heart and postsynaptic adrenergic receptors to greater concentrations of norepinephrine, which in turn causes desensitization/downregulation of $\beta$-adrenergic receptors,

Received Dec. 16, 2016; revision accepted Feb. 15, 2017.

For correspondence or reprints contact: Albert J. Sinusas, Section of Cardiovascular Medicine, Yale University School of Medicine, P.O. Box 208017, Dana 3, New Haven, CT 06520-8017.

E-mail: albert.sinusas@yale.edu

Published online Feb. 23, 2017.

COPYRIGHT (c) 2017 by the Society of Nuclear Medicine and Molecular Imaging. DOI: 10.2967/jnumed.116.186130 cardiac remodeling, and worsening of $\mathrm{HF}$ and prognosis (10). Importantly, in vivo noninvasive assessment of sympathetic innervation with SPECT and PET imaging using radiolabeled analogs of norepinephrine have provided valuable prognostic information in patients with HFrEF beyond currently available biomarkers, such as BNP and LV ejection fraction. The most commonly used SPECT tracer to assess cardiac sympathetic innervation is ${ }^{123} \mathrm{I}-$ metaiodobenzylguanadine ( $\left.{ }^{123} \mathrm{I}-\mathrm{mIBG}\right)$, whereas the most commonly used PET tracer is ${ }^{11} \mathrm{C}$-hydroxyephedrine $\left({ }^{11} \mathrm{C}\right.$-HED). Both of these tracers are norepinephrine radioanalogs, and their uptake primarily represents presynaptic nerve function (or density) in the heart.

LV diastolic dysfunction is a characteristic finding in patients with $\mathrm{HFpEF}$, and increasing severity of diastolic dysfunction is related to HF progression and a worse prognosis in these patients $(13,14)$. Several lines of evidence suggest that dysregulated SNS activity plays an important role in the pathophysiology of HFpEF, particularly in the development of diastolic dysfunction. Preclinical studies show that mimicking an elevation of SNS activity via isoproterenol administration leads to diastolic dysfunction, accompanied by increased myocardial stiffening, fibrosis, and LV hypertrophy (15). Grassi et al. (16) observed that patients with diastolic dysfunction and hypertension display higher SNS activity (e.g., muscle sympathetic nerve activity [MSNA]) and abnormal baroreflex modulation compared with hypertensive patients without diastolic dysfunction, and both these groups show higher MSNA than age-matched controls. In these studies, MSNA was significantly and inversely related to various transthoracic echocardiographic indices of diastolic dysfunction (e.g., E/A wave ratio, deceleration time, and isovolumic relaxation time). Other human studies have observed similar findings with respect to the relationship between SNS activity and HFpEF (15), although many of these studies are limited by the small sample sizes, and not all of these studies report on diastolic parameters. Interestingly, albeit in a limited number of studies, it has been shown that indices of sympathetic presynaptic nerve function derived from planar ${ }^{123}$ I-mIBG scintigraphy correlate with the severity of diastolic dysfunction, exercise capacity, LV remodeling, response to therapy, and HF severity and are able to predict adverse cardiovascular events in patients with HFpEF (17-19). More specifically, the ${ }^{123} \mathrm{I}-\mathrm{mIBG}$ heart-to-mediastinal ratio and ${ }^{123} \mathrm{I}-\mathrm{mIBG}$ washout rate have proved to be the most useful indices in these studies. Indeed, the small number of patients, the use of different definitions of HFpEF and diastolic dysfunction, and the application of only semiquantitative analyses of ${ }^{123} \mathrm{I}-\mathrm{mIBG}$ planar scintigraphy have limited the generalizability and strength of these findings. 
In this current issue of The Journal of Nuclear Medicine, Aikawa et al. (20) performed PET imaging with ${ }^{11} \mathrm{C}$-HED in 41 subjects with HFpEF (LV ejection fraction $\geq 40 \%$ ) and 12 normal volunteers in an effort to establish the relationship between impaired cardiac SNS innervation and LV diastolic dysfunction in HFpEF patients. As outlined above, although previous studies have already reported similar relationships with ${ }^{123} \mathrm{I}-\mathrm{mIBG}$ planar scintigraphic indices in HFpEF patients $(18,19)$, the novelty of this study relates to use of ${ }^{11} \mathrm{C}$-HED PET imaging, which allows for improved global and regional quantification of SNS innervation due to better spatiotemporal resolution and more quantitative image analysis than conventional ${ }^{123} \mathrm{I}-\mathrm{mIBG}$ planar imaging. In this study, ${ }^{11} \mathrm{C}$-HED-derived indices of cardiac sympathetic function were related to transthoracic echocardiographic indices of diastolic dysfunction as established in the 2009 recommendations of the American Society of Echocardiography (ASE) and European Association of Echocardiography (EAE) (21). Global cardiac SNS innervation was expressed using the semiquantitative, retention index (RI $\left[\mathrm{min}^{-1}\right]$ ), and a 17-segement model was used to calculate RI in different myocardial regions. The heterogeneity of cardiac SNS innervation was determined by the coefficient of variation among the RIs for each of the 17 segments. Aikawa et al. reported that ${ }^{11} \mathrm{C}-\mathrm{HED}$ uptake was globally reduced and more heterogeneous in $\mathrm{HFpEF}$ patients than in age-matched controls and that patients with more severe diastolic dysfunction (grade 2-3) had a greater reduction in global ${ }^{11} \mathrm{C}$-HED uptake with more heterogeneous uptake than patients with less severe diastolic dysfunction (grade 0-1). Importantly, because myocardial ischemia and infarction have been shown to independently influence ${ }^{11} \mathrm{C}-\mathrm{HED}$ uptake, the investigators also compared global and heterogeneous uptake of ${ }^{11} \mathrm{C}$-HED in patients with a nonischemic etiology of HF and found that only global RI, but not the variance of RI, was significantly different between grades of diastolic dysfunction among the HFpEF patients.

Although this study is novel and clinically relevant, there are several limitations that are worth mentioning. First, and most importantly, these authors define diastolic dysfunction based on the 2009 recommendations of the ASE and European Association of Cardiovascular Imaging (EACVI, formerly EAE) despite the recent publication of new guidelines by these same organizations (22). As shown in the supplemental materials for the Aikawa et al. (20) article, when the authors used the most recent guidelines for diastolic function assessment, $70 \%$ of patients were reclassified, and global RI became similar among the HFpEF patients despite differences in the severity of diastolic dysfunction. It is possible that the reclassification of patients using the current guidelines led to a nonsignificant finding between different grades of diastolic dysfunction, because the recently recommended algorithm does not explicitly describe how to classify patients with atrial fibrillation. Consequently, the number of patients included in the analysis was reduced. However, it is worth noting that the new guidelines do provide alternatives for grading LV diastolic dysfunction in special populations, including atrial fibrillation. Notwithstanding the ambiguity in the new algorithm, the classification of diastolic dysfunction in the current article is at odds with the 2009 ASE/EACVI algorithm. Specifically, it appears that some patients were classified as having diastolic dysfunction despite having normal left atrial volumes (Table 2 in Aikawa et al. (20)). However, the 2009 guidelines of the ASE/EACVI suggest that an enlarged left atrial volume index $\left(\geq 34 \mathrm{~mL} / \mathrm{m}^{2}\right)$ is an absolute criterion for diastolic dysfunction, especially for grades 2 and 3 dysfunction. Clearly, future studies exploring the relationship between ${ }^{11} \mathrm{C}$-HED uptake and diastolic dysfunction in $\mathrm{HFpEF}$ will need to use current criteria for grading diastolic dysfunction. On the other hand, the relation between dysfunctional SNS innervation to diastolic dysfunction may not be as important as the relationship between global ${ }^{11} \mathrm{C}$-HED uptake to cardiovascular prognosis and mortality in these HFpEF patients. Along these lines, decreased global ${ }^{11} \mathrm{C}$-HED RI is independently predictive of worse cardiovascular prognosis and all-cause mortality in patients with $\operatorname{HFrEF}(23,24)$. More relevant to the topic at hand, other studies have reported that the ${ }^{123} \mathrm{I}$-mIBG heart-to-mediastinal ratio and washout rate can discriminate between HF severity and can predict worse prognosis and future cardiac events in HFpEF patients $(18,19)$.

Although useful for a general assessment of the cardiac presynaptic uptake-1 mechanism, ${ }^{11} \mathrm{C}$-HED cannot distinguish between denervated myocardium and myocardium with intact neurons but with dysfunctional uptake-1 (dysinnervated myocardium). ${ }^{11} \mathrm{C}-\mathrm{HED}$ uptake may be reduced through various mechanisms including SNS presynaptic denervation, impairments in the energy required for proper norepinephrine transporter function (i.e., ischemia), a downregulation of norepinephrine transporter receptors, and competitive inhibition of ${ }^{11} \mathrm{C}$-HED for norepinephrine transporter receptor sites by excess synaptic norepinephrine $(25,26)$. The mechanism leading to reduced ${ }^{11} \mathrm{C}-\mathrm{HED}$ uptake may vary along the continuum of HFpEF progression, and may well also vary depending on the etiology of HFpEF (e.g., amyloidosis vs. hypertension). Although SNS presynaptic activity can be assessed semiquantitatively with ${ }^{11} \mathrm{C}$ HED (i.e., ${ }^{11} \mathrm{C}-\mathrm{HED}$ washout), the consecutive use of multiple PET adrenergic tracers with different uptake and storage kinetics may also help to elucidate mechanisms of SNS innervation impairments in HFpEF patients with differing severities and underlying pathophysiologic disease processes. Along these lines, the consecutive use of ${ }^{11} \mathrm{C}$-HED, ${ }^{11} \mathrm{C}$-epinephrine, and ${ }^{11} \mathrm{C}$-phenlyphrine has provided valuable mechanistic information of sympathetic innervation impairments in postinfarct models and in the pattern of sympathetic neuronal regeneration in cardiac transplantation patients $(27,28)$.

The benefit of performing PET imaging compared with SPECT imaging is related to the higher spatiotemporal resolution of PET imaging, and more established methods for kinetic modeling and absolute quantification of radiotracer uptake. Therefore, an important limitation to this study is the use of the semiquantitative RI. Along these lines, it has been debated that the ${ }^{11} \mathrm{C}$-HED RI may not be sensitive enough to detect mild-to-moderate nerve loss (29). Recently, a fully quantitative kinetic model has been established for ${ }^{11} \mathrm{C}$-HED (30) and confirmed that the quantitative parameter, volume of distribution, is nonlinearly related to SUV and RI, especially in the setting of lower radiotracer uptake. Interestingly, this same group has developed methods for absolute quantification of ${ }^{11} \mathrm{C}$-HED uptake using dynamic imaging that only require venous blood sampling, given that venous-to-arterial metabolite transformation corrections are applied (31). Deriving volume of distribution values noninvasively from ${ }^{11} \mathrm{C}$-HED PET studies will be beneficial for fully elucidating the relationship between cardiac denervation and different grades of LV diastolic dysfunction in HFpEF patients, particularly if the differences in tracer uptake are subtle. On the other hand, quantitative ${ }^{123} \mathrm{I}$-mIBG SPECT imaging may provide an alternative to ${ }^{11} \mathrm{C}$-HED PET imaging in $\mathrm{HFpEF}$ patients, especially at sites without access to a cyclotron. Our group has recently reported a method for absolute quantitative evaluation of dynamic ${ }^{123} \mathrm{I}-\mathrm{mIBG}$ SPECT images using a high-resolution and high-sensitivity dedicated 
cardiac cadmium zinc telluride imaging system (Discovery NM/CT 570c; GE Healthcare) (32). This method uses a reversible 2-tissuecompartment model and population-based corrections for blood-toplasma ratio and metabolites (32). Therefore, the quantitative evaluation of the SNS may also be feasible with ${ }^{123}$ I-mIBG using appropriate kinetic models and newer high-resolution and high-sensitivity dedicated cardiac cadmium zinc telluride imaging systems.

In summary, the study by Aikawa et al. (20) was the first to show a relationship between impaired cardiac SNS innervation noninvasively assessed with ${ }^{11} \mathrm{C}$-HED PET imaging and the severity of diastolic dysfunction using transthoracic echocardiography in HFpEF patients. Despite the methodologic limitations, this study provides the foundation for further exploration of the role of the SNS in HFpEF. PET imaging of SNS function with ${ }^{11} \mathrm{C}-\mathrm{HED}$ may provide additional prognostic information over available biomarkers and imaging of cardiac diastolic function with echocardiography. Future studies should use methodologies that can provide an absolute quantification of SNS function and should use current guidelines with respect to grading diastolic dysfunction in HFpEF patients. Quantitative ${ }^{123}$ I-mIBG SPECT imaging may provide an alternative to ${ }^{11} \mathrm{C}$-HED PET imaging in $\mathrm{HFpEF}$ patients, given that ${ }^{123} \mathrm{I}$-mIBG is a readily available Food and Drug Administration-approved pharmaceutical and quantitative analysis of dynamic ${ }^{123} \mathrm{I}-\mathrm{mIBG}$ SPECT imaging is now possible (32). The application of quantitative ${ }^{123}$ I-mIBG SPECT imaging to assess SNS innervation in HF patients may provide an opportunity for centers that do not have access to a costly, onsite cyclotron for production of ${ }^{11} \mathrm{C}$-HED to explore the clinically relevant role of cardiac SNS dysinnervation in the prognosis of HFpEF patients.

\section{DISCLOSURE}

No potential conflict of interest relevant to this article was reported.

\section{REFERENCES}

1. Roger VL. Epidemiology of heart failure. Circ Res. 2013;113:646-659.

2. Bhatia RS, Tu JV, Lee DS, et al. Outcome of heart failure with preserved ejection fraction in a population-based study. $N$ Engl J Med. 2006;355:260-269.

3. Bursi F, Weston SA, Redfield MM, et al. Systolic and diastolic heart failure in the community. JAMA. 2006;296:2209-2216.

4. Ponikowski P, Voors AA, Anker SD, et al. ESC Guidelines for the diagnosis and treatment of acute and chronic heart failure. Eur Heart J. 2016;37:2129-2200.

5. Yancy CW, Jessup M, Bozkurt B, et al. ACCF/AHA guideline for the management of heart failure: a report of the American College of Cardiology Foundation/ American Heart Association Task Force on Practice Guidelines. Circulation. 2013;128:1810-1852.

6. Udelson JE. Heart failure with preserved ejection fraction. Circulation. 2011;124:e540-e543.

7. Gladden JD, Linke WA, Redfield MM. Heart failure with preserved ejection fraction. Pflügers Archiv. 2014;466:1037-1053.

8. Owan TE, Hodge DO, Herges RM, Jacobsen SJ, Roger VL, Redfield MM. Trends in prevalence and outcome of heart failure with preserved ejection fraction. $N$ Engl J Med. 2006;355:251-259.

9. Yancy CW, Jessup M, Bozkurt B, et al. ACC/AHA/HFSA focused update on new pharmacological therapy for heart failure: an update of the 2013 ACCF/AHA guideline for the management of heart failure - a report of the American College of Cardiology/American Heart Association Task Force on Clinical Practice
Guidelines and the Heart Failure Society of America. J Am Coll Cardiol. 2016;68:1476-1488.

10. Haider N, Baliga RR, Chandrashekhar Y, Narula J. Adrenergic excess, hNET1 down-regulation, and compromised $\mathrm{mIBG}$ uptake in heart failure: poverty in the presence of plenty. JACC Cardiovasc Imaging. 2010;3:71-75.

11. Cohn JN, Levine TB, Olivari MT, et al. Plasma norepinephrine as a guide to prognosis in patients with chronic congestive heart failure. N Engl J Med. 1984;311:819-823.

12. Kaye DM, Lambert GW, Lefkovits J, Morris M, Jennings G, Esler MD. Neurochemical evidence of cardiac sympathetic activation and increased central nervous system norepinephrine turnover in severe congestive heart failure. $\mathrm{J} \mathrm{Am}$ Coll Cardiol. 1994;23:570-578.

13. Ohtani T, Mohammed SF, Yamamoto K, et al. Diastolic stiffness as assessed by diastolic wall strain is associated with adverse remodelling and poor outcomes in heart failure with preserved ejection fraction. Eur Heart J. 2012;33:17421749 .

14. Redfield MM, Jacobsen SJ, Burnett JC Jr, Mahoney DW, Bailey KR, Rodeheffer RJ. Burden of systolic and diastolic ventricular dysfunction in the community: appreciating the scope of the heart failure epidemic. JAMA. 2003;289:194-202.

15. Verloop WL, Beeftink MM, Santema BT, et al. A systematic review concerning the relation between the sympathetic nervous system and heart failure with preserved left ventricular ejection fraction. PLoS One. 2015;10:e0117332.

16. Grassi G, Seravalle G, Quarti-Trevano F, et al. Sympathetic and baroreflex cardiovascular control in hypertension-related left ventricular dysfunction. Hypertension. 2009;53:205-209.

17. Kasama S, Toyama $\mathrm{T}$, Kumakura $\mathrm{H}$, et al. Effects of candesartan on cardiac sympathetic nerve activity in patients with congestive heart failure and preserved left ventricular ejection fraction. J Am Coll Cardiol. 2005;45:661-667.

18. Sugiura M, Yamamoto K, Takeda Y, et al. The relationship between variables of 123-I-metaiodobenzylguanidine cardiac imaging and clinical status of the patients with diastolic heart failure. Int J Cardiol. 2006;113:223-228.

19. Katoh S, Shishido T, Kutsuzawa D, et al. Iodine-123-metaiodobenzylguanidine imaging can predict future cardiac events in heart failure patients with preserved ejection fraction. Ann Nucl Med. 2010;24:679-686.

20. Aikawa T, Naya M, Obara M, et al. Impaired myocardial sympathetic innervation is associated with diastolic dysfunction in heart failure with preserved ejection fraction: ${ }^{11}$ C-hydroxyephedrine PET study. J Nucl Med. 2017;58:784-790.

21. Nagueh SF, Appleton CP, Gillebert TC, et al. Recommendations for the evaluation of left ventricular diastolic function by echocardiography. J Am Soc Echocardiogr. 2009;22:107-133.

22. Nagueh SF, Smiseth OA, Appleton CP, et al. Recommendations for the evaluation of left ventricular diastolic function by echocardiography: an update from the American Society of Echocardiography and the European Association of Cardiovascular Imaging. J Am Soc Echocardiogr. 2016;29:277-314.

23. Fujita W, Matsunari I, Aoki H, Nekolla SG, Kajinami K. Prediction of all-cause death using ${ }^{11} \mathrm{C}$-hydroxyephedrine positron emission tomography in Japanese patients with left ventricular dysfunction. Ann Nucl Med. 2016;30:461-467.

24. Pietilä M, Malminiemi K, Ukkonen H, et al. Reduced myocardial carbon-11 hydroxyephedrine retention is associated with poor prognosis in chronic heart failure. Eur J Nucl Med. 2001;28:373-376.

25. Chen GP, Tabibiazar R, Branch KR, Link JM, Caldwell JH. Cardiac receptor physiology and imaging: an update. J Nucl Cardiol. 2005;12:714-730.

26. Fallavollita JA, Canty JM Jr. Dysinnervated but viable myocardium in ischemic heart disease. J Nucl Cardiol. 2010;17:1107-1115.

27. Bravo PE, Lautamäki R, Carter D, et al. Mechanistic insights into sympathetic neuronal regeneration multitracer molecular imaging of catecholamine handling after cardiac transplantation. Circ Cardiovasc Imaging. 2015;8:e03507.

28. Lautamaki R, Sasano T, Higuchi T, et al. Multiparametric molecular imaging provides mechanistic insights into sympathetic innervation impairment in the viable infarct border zone. J Nucl Med. 2015;56:457-463.

29. Raffel DM, Koeppe RA, Jung Y-W, et al. Quantification of cardiac sympathetic nerve density with $\mathrm{N}-{ }^{11} \mathrm{C}$-guanyl-meta-octopamine and tracer kinetic analysis. $J$ Nucl Med. 2013;54:1645-1652.

30. Harms HJ, de Haan S, Knaapen P, et al. Quantification of [11 C]-metahydroxyephedrine uptake in human myocardium. EJNMMI Res. 2014;4:52.

31. Harms HJ, Huisman MC, Rijnierse MT, et al. Non-invasive quantification of myocardial ${ }^{11} \mathrm{C}$-meta-hydroxyephedrine kinetics. J Nucl Med. 2016;57:1376-1381.

32. Wu J, Lin S-f, Gallezot J-D, et al. Quantitative analysis of dynamic ${ }^{123}$ I-mIBG SPECT imaging data in healthy humans with a population-based metabolite correction method. J Nucl Med. 2016;57:1226-1232. 